\title{
Un gioco-indagine per scoprire teoremi di geometria
}

\section{An inquiring-game activity to discover geometry theorems}

\author{
Carlotta Soldano $^{\star}$ e Gaetano Di Caprio ${ }^{\circ}$ \\ ^Dipartimento di Filosofia e Scienze dell’Educazione - Università degli Studi di Torino, Italia \\ ${ }^{\circ}$ Istituto di Istruzione Superiore Erasmo da Rotterdam - Nichelino (Torino), Italia
}

Sunto / Il presente articolo illustra un'attività di gioco-indagine progettata all'interno di GeoGebra a partire da un teorema di geometria euclidea, riformulato secondo l'approccio della Logica dell'Indagine (Hintikka, 1999). L'obiettivo della ricerca è analizzare il contributo che la Logica dell'Indagine, implementata all'interno di ambienti di geometria dinamica, può offrire nell'educazione degli studenti alla scoperta e alla riorganizzazione logica della loro conoscenza matematica. L'attività didattica, sperimentata nell'anno scolastico 2018-2019 in una classe seconda liceo scientifico, è stata organizzata in tre fasi: il gioco, l'indagine e la discussione di classe. L'analisi dei processi investigativi e argomentativi degli studenti, unitamente all'analisi a priori dell'attività, intende evidenziare come questo tipo di percorso possa avvicinare gli studenti ad intuire ciò che definiamo il lavoro "vero" del matematico, che consiste nell'esplorare, congetturare, verificare e dimostrare teoremi.

Parole chiave: Attività di gioco-indagine; ragionamento regressivo; logica dell'indagine; gioco semantico; relazioni geometriche.
Abstract / This article illustrates an inquiring-game activity designed within GeoGebra starting from a Euclidean geometry theorem reformulated according to the approach of the Logic of Inquiry (Hintikka, 1999). The aim of the research is to analyze the contribution that the Logic of Inquiry, implemented within dynamic geometry environments, can offer in the education of students to the discovery and logical reorganization of their mathematical knowledge. The didactic activity, experimented in the school year 2018-2019 in a secondary school scientific class, was organized in three phases: the game, the investigation and the class discussion. The analysis of students' inquiring processes and argumentation, together with the a priori analysis of the activity, intends to highlight how this type of activity can bring students closer to what we define as the "true" work of the mathematician, which consists in exploring, conjecturing, verifying and proving theorems.

Keywords: Inquiring-game activity; regressive reasoning; logic of inquiry; semantical games; geometric relationship.

Come viene messo in luce dal MIUR nel "Regolamento recante norme in materia di adempimento dell'obbligo di istruzione", tra le finalità dell'asse culturale matematico c'è anche l'acquisizione delle abilità necessarie «per seguire e vagliare la coerenza logica delle argomentazioni proprie e altrui in molteplici contesti di indagine conoscitiva e di decisione» (MIUR, 2007). In particolare, le competenze argomentative costituiscono la base per preparare gli studenti alla dimostrazione, attività che contraddistingue il pensiero matematico maturo. Garuti, Boero, Lemut e Mariotti (1996) sostengono che in alcuni casi possa esistere una "continuità cognitiva" tra i processi di produzione di congetture e la costruzione di dimostrazioni. Essa si verifica se gli studenti, nella fase di produzione della congettura, innescano un'intensa attività 
argomentativa riguardo la plausibilità delle loro affermazioni e, nella fase dimostrativa, si ricollegano agli argomenti prodotti nella fase di produzione della congettura, riorganizzandoli secondo catene deduttive.

Gli ambienti di geometria dinamica si rivelano particolarmente efficaci nella costruzione di enunciati e significati matematici perché permettono agli studenti di effettuare intense attività di esplorazione che favoriscono la produzione di congetture e argomentazioni. Le esplorazioni avvengono attraverso lo strumento di trascinamento che consiste nel muovere un elemento della figura sullo schermo ottenendo in questo modo altri esemplari della stessa famiglia di figure. Trascinando un elemento geometrico dinamico è possibile distinguere "punti (e in generale oggetti) liberi cioè costruiti come nuovi oggetti sullo schermo - da punti (oggetti) dipendenti cioè costruiti a partire da altri elementi, con cui sono messi in relazione» (Antonini \& Baccaglini Frank, 2015, p. 260). Attraverso questa esperienza è possibile notare gli invarianti di trascinamento che consistono in proprietà che definiscono e caratterizzano gli oggetti geometrici o in enunciati di tipo condizionale, ossia enunciati del tipo "se... allora...". Quando viene trascinata una figura all'interno degli ambienti di geometria dinamica, non sempre si conservano le proprietà che definiscono la figura stessa. Le costruzioni dinamiche le cui proprietà si conservano durante il trascinamento, vengono dette costruzioni robuste (Healy, 2000; Laborde, 2005), le costruzioni che si "deformano" perdendo le loro proprietà caratteristiche, vengono dette soft. Nell'insegnamento-apprendimento della geometria, gli ambienti di geometria dinamica possono essere utilizzati per la progettazione di attività in cui viene chiesto agli studenti di esplorare e congetturare proprietà geometriche, tra cui quelle espresse in forma condizionale, osservate in fase esplorativa come invarianti di trascinamento. A tal fine, generalmente, vengono proposti agli studenti problemi aperti (Arsac, 1999), ossia problemi che non svelano la soluzione o la risposta, ma in cui viene descritta una configurazione e viene chiesto agli studenti di produrre congetture riguardanti le relazioni tra gli elementi della configurazione descritta dal problema. Queste esperienze si rivelano particolarmente utili perché avvicinano gli studenti alla doppia natura figurale e concettuale degli oggetti geometrici (Fischbein, 1993). Ogni oggetto geometrico è il prodotto della sua definizione e possiede una natura concettuale ma allo stesso tempo possiede anche una natura figurale che consiste nella sua rappresentazione grafica. Questa duplice natura rende gli oggetti geometrici dei concetti figurali (Fischbein, 1993). È fondamentale che gli studenti comprendano e armonizzino i due aspetti, per non lasciarsi trarre in inganno da elementi grafici presenti nella rappresentazione dell'oggetto geometrico e per sviluppare un pensiero produttivo in geometria.

In questo studio, anziché proporre agli studenti problemi aperti, abbiamo scelto di innescare l'esplorazione e la produzione di congetture e argomentazioni attraverso un gioco semantico appositamente progettato a partire dall'enunciato di un teorema di geometria. Partendo da una situazione empirica di gioco all'interno dell'ambiente di geometria dinamica GeoGebra, gli studenti vengono guidati nella scoperta dell'enunciato del teorema e nella sua dimostrazione, intrecciando aspetti pratici-empirici con gli aspetti teorici e logici. Si tratta quindi di un'attività didattica in cui aspetti logici e geometrici si intrecciano inscindibilmente nella scoperta e costruzione della conoscenza matematica. 


\section{Quadro teorico di riferimento}

In questa ricerca, la Logica dell'Indagine e la Semantica della Teoria dei Giochi, sviluppate dal logico e filosofo finlandese Jaakko Hintikka (1929-2015), costituiscono il quadro teorico con cui è stato progettato il gioco attraverso cui sono stati analizzati i protocolli degli studenti.

Secondo la Semantica della Teoria dei Giochi, è possibile stabilire la verità degli enunciati matematici attraverso giochi semantici, ossia sfide tra un verificatore che deve mostrare la verità di un enunciato e un falsificatore che deve cercare di stabilirne la falsità. Ci limiteremo a considerare giochi semantici associati ad enunciati del tipo "per ogni $x$, esiste $y$ tale che $S(x, y)$ ». Per mostrare la verità di questo tipo di enunciato è sempre possibile immaginare un gioco tra un falsificatore che controlla la variabile $x$ e un verificatore che controlla la variabile $y$. II falsificatore sceglie un valore della variabile $x$ e il verificatore deve trovare il valore della variabile $y$ che rende vera $S(x, y)$. In base alla semantica della teoria dei giochi, se esiste una strategia vincente per il verificatore del gioco allora l'enunciato è vero, altrimenti è falso. Grazie a tale strategia, il verificatore ha la possibilità di vincere qualsiasi sia il valore di $x$ proposto dal falsificatore. Per chiarire la dinamica di gioco, la illustreremo attraverso un semplice esempio. Consideriamo l'enunciato «per ogni numero naturale $n$ esiste un numero naturale $m$ tale che $m-3 n=1$ ». Per stabilire la verità di questo enunciato possiamo immaginare un gioco in cui un falsificatore sceglie il valore di $n$ e un verificatore deve far vedere che per quel valore di n esiste un numero naturale $m$ che gli permette di vincere il gioco. Se il falsificatore sceglie ad esempio $n=7$, il verificatore mostrerà che con $m=22$ vince il gioco perché $22-3 \cdot 7=1$. Dal momento che l'insieme dei numeri naturali è chiuso per moltiplicazione e addizione, il verificatore ha la possibilità di vincere sempre. Infatti, basterà scegliere il numero consecutivo del triplo del numero scelto dal falsificatore per verificare l'enunciato su qualsiasi numero naturale scelto dal falsificatore. Essendo un gioco competitivo, il falsificatore cercherà di mettere in difficoltà il verificatore, proponendogli ad esempio valori di $n$ molto grandi. Dal momento che esiste una strategia vincente per il verificatore del gioco, I'enunciato è vero secondo la semantica della teoria dei giochi.

La Logica dell'Indagine è una logica diversa dalla logica classica, si tratta di una logica interrogativa, basata sulla dialettica della domanda e della risposta. Essa, accanto al ragionamento di tipo deduttivo, ammette anche forme di ragionamento ampliativo, attraverso le quali nuovi elementi, non contenuti all'interno delle premesse del ragionamento, possono essere introdotti nel discorso. Queste forme di ragionamento sono il risultato di abduzione (Peirce, 1960). Per comprendere l'abduzione e la sua differenza rispetto alla deduzione, proponiamo un esempio classico, illustrato da Peirce stesso (Peirce, 1960, p. 372). Immaginiamo di sapere che una certa borsa è piena di fagioli bianchi e di vedere per terra dei fagioli bianchi, possiamo allora inferire "Questi fagioli provengono da quella borsa». Questa inferenza è un'abduzione, ossia un'ipotesi che permette di spiegare i fatti osservati. Schematicamente potremmo descriverla cosi:

$A$ : Questi fagioli sono bianchi.

$B$ : Questi fagioli vengono da quella borsa.

So che $B \rightarrow A$ (cioè che se questi fagioli vengono da quella borsa allora questi fagioli sono bianchi) e che $A$ è vera (cioè che questi fagioli sono bianchi); inferisco che $B$ sia vera (ossia che questi fagioli vengono da quella borsa). 
Si tratta di un ragionamento diverso dalla deduzione che parte dall'ipotesi $B$ e dalla conoscenza che $B \rightarrow A$ e conclude $A$.

In un ragionamento deduttivo a partire dalla regola $(B \rightarrow A)$ e dal caso $(B)$ si ricava il risultato $(A)$, in un ragionamento abduttivo a partire dalla regola $(B \rightarrow A)$ e dal risultato $(A)$ si ipotizza il caso $(B)$. Ovviamente un'abduzione ha solo un certo grado di probabilità di essere vera, quei fagioli bianchi potrebbero provenire dal sacchetto di un passante che li ha persi camminando.

Le abduzioni sono forme di ragionamento utilizzate dai detective nelle loro indagini. Immaginando di dover analizzare la scena di un crimine alla ricerca dall'assassino, il metodo investigativo, generalmente, non procede per via esclusivamente deduttiva, ma prevederà l'alternarsi e l'intrecciarsi di ragionamenti di tipo regressivo (Barbero \& Gómez-Chacón, 2018), ossia partendo da ciò che si sa si procede "all'indietro" alla ricerca dei fatti da cui discende, e di tipo progressivo, ossia "in avanti" dai fatti alle loro conseguenze. I passi regressivi nel ragionamento possono essere il frutto di abduzioni. Ragionamenti progressivi e regressivi sono il cuore di ciò che Hintikka chiama la Logica dell'Indagine e saranno oggetto di analisi dello studio di caso che presenteremo in questo articolo.

\section{Merodolosat}

La Logica dell'Indagine ha costituito la base per la progettazione di un'attività di gioco-indagine all'interno dell'ambiente di geometria dinamica GeoGebra. Tale attività è stata oggetto di una sperimentazione didattica effettuata in una scuola secondaria di secondo grado ${ }^{1}$, con la finalità di studiare l'intreccio tra processi d'indagine e processi dimostrativi secondo l'approccio della logica dell'Indagine. L'attività didattica, progettata e sperimentata nell'anno scolastico 2018-2019, è stata organizzata in tre fasi: il gioco prevede una sfida in GeoGebra tra due studenti che, seguendo le regole riportate in una scheda, ricoprono di volta in volta il ruolo di verificatore e falsificatore e utilizzano un file GeoGebra appositamente progettato come terreno di gioco. II design è ispirato ai giochi semantici di Hintikka (1998) e, potenzialmente, può essere implementato su qualsiasi enunciato matematico esprimibile nella forma "per ogni... esiste...". All'interno di questo contesto, le variabili controllate dai giocatori sono punti di figure dinamiche che il verificatore e il falsificatore trascinano al fine di raggiungere i rispettivi obiettivi. In questa fase gli studenti devono scoprire la strategia vincente per il verificatore del gioco, ossia la condizione geometrica attraverso cui guidare le proprie mosse per vincere sempre. L'indagine è guidata da apposite domande volte a scoprire, formulare, dimostrare e generalizzare l'enunciato del teorema su cui si basa il gioco. In questa fase l'ambiente di geometria dinamica potrà essere utilizzato come supporto nella formulazione e validazione delle congetture. Infine, la discussione di classe prevede la condivisione delle scoperte e delle riflessioni degli studenti e l'istituzionalizzazione del sapere matematico su cui è basata l'attività.

La sperimentazione è stata svolta nelle ore di matematica curriculari in una settimana di aprile 2019, coinvolgendo sedici studenti di seconda liceo scientifico (grado

1. La scuola secondaria di secondo grado italiana corrisponde ai gradi 9-13, comprende quindi la quarta media e la scuola media superiore svizzere. 
10). Quattordici di questi studenti conoscevano già le dinamiche di questi giochi perché erano stati coinvolti in una sperimentazione analoga l'anno precedente [per approfondimenti vedere Soldano e Sabena (2019)]. La durata della sperimentazione è stata di quattro ore, articolate come segue nell'arco di tre giorni:

- Primo giorno: attività introduttiva avente l'obiettivo di presentare un teorema matematico (teorema della divisione euclidea) attraverso il gioco della corsa a 20 (Brousseau, 1997). Durata complessiva: un'ora.

- Secondo giorno: attività di gioco-indagine in GeoGebra. Durata complessiva: due ore, di cui circa trenta minuti dedicati al gioco e il restante tempo alla scheda di riflessione sul gioco.

- Terzo giorno: commenti alle risposte date dagli studenti nella scheda di riflessione sul gioco e sistematizzazione della conoscenza matematica. Durata complessiva: un'ora.

I dati raccolti sono costituiti da un video di una coppia di studenti mentre svolge I'attività di gioco-indagine, il video della discussione di classe condotta il terzo giorno e i protocolli di tutti gli studenti.

All'interno degli ambienti di geometria dinamica, grazie alla possibilità di trascinare gli oggetti geometrici ed osservare gli effetti di questi movimenti, è possibile favorire l'esplorazione, la formulazione di congetture e la loro generalizzazione. Attraverso la progettazione di opportune situazioni didattiche, gli studenti possono sentire l'esigenza di dimostrare quanto osservato o scoperto per spiegarselo o per convincere sé stessi e/o gli altri. Dal punto di vista della ricerca, I'obiettivo dell'attività consiste nel cercare di armonizzare il divario epistemico e cognitivo messo in luce in letteratura riguardo al passaggio dall'esplorazione empirica alla dimostrazione teorica. Dal punto di vista didattico l'attività ha l'obiettivo di promuovere negli studenti il bisogno di dimostrare partendo da un contesto di gioco e investigazione, che si suppone possa far nascere tale bisogno. La nostra ipotesi è che la dinamica verificatore/falsificatore fornisca agli studenti una base concreta su cui elaborare riflessioni teoriche.

\section{L'attività di gioco-indagine}

L'attività di gioco-indagine che è oggetto del presente articolo prende ispirazione dal problema presentato al Rally Matematico Transalpino negli anni 2000 riportato in Figura 1:

Questo è il giardino del signor Torquato:

Nella parte grigia egli ha piantato fiori e ha seminato a prato la parte bianca.

Il signor Torquato osserva il suo giardino e si chiede:

"Sarà maggiore la parte con i fiori o quella con il prato?"

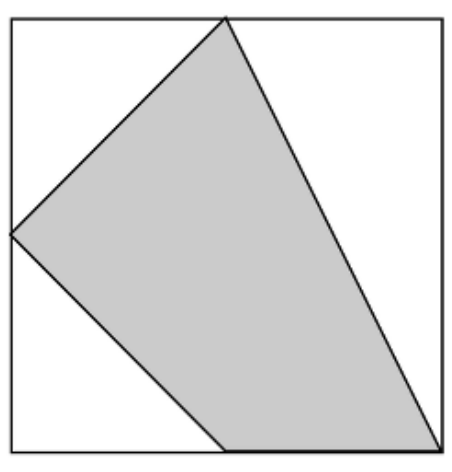


Scomponendo la figura in triangoli a due a due congruenti, è possibile stabilire l'equivalenza delle due aree messe a confronto.

Generalizzando questo problema, abbiamo proposto agli studenti un'attività di gioco-indagine il cui obiettivo è la scoperta della condizione necessaria e sufficiente da cui deriva l'equivalenza tra le aree. La Figura 2 riporta la schermata del gioco implementato in GeoGebra:

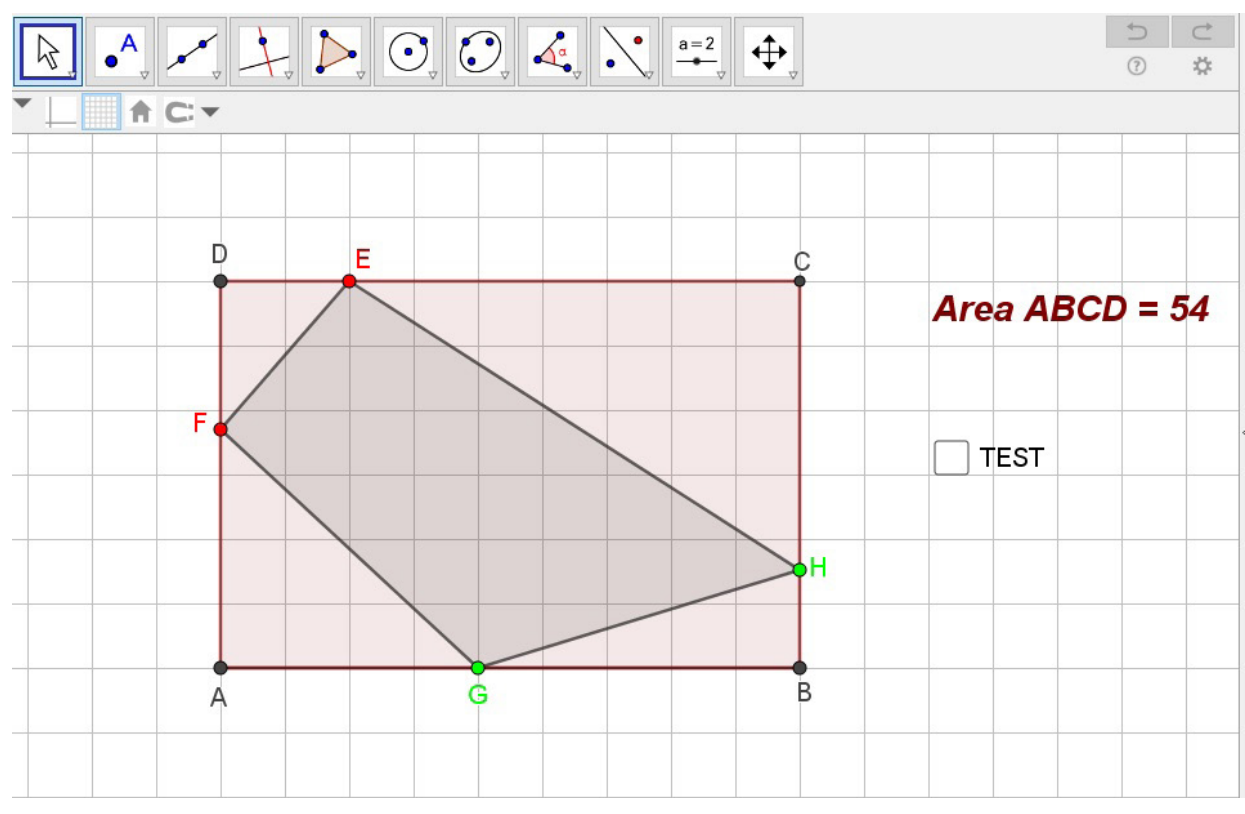

A differenza del quesito del Rally Matematico Transalpino, il gioco richiede di variare il quadrilatero inscritto $E F G H$ affinché la sua area sia pari alla metà di quella del rettangolo $A B C D$. I vertici di $E F G H$ sono oggetti trascinabili sui rispettivi lati di $A B C D$ su cui sono definiti. Quando (e solo quando) almeno una coppia di vertici opposti è allineata parallelamente ad un lato del rettangolo si verifica che l'area di $E F G H$ è la metà dell'area di $A B C D$. Questa condizione rappresenta la strategia vincente che garantisce al verificatore di vincere il gioco qualsiasi sia la configurazione dei punti $E$ e $F$ proposta dal falsificatore.

Riportiamo di seguito le istruzioni del gioco date agli studenti:

- All'interno della vostra coppia stabilite un verificatore e un falsificatore. Ogni sfida è costituita da due mosse e da un test.

- La prima mossa è quella del falsificatore che muovendo i punti $E$ ed $F$ deve fare in modo che l'area di $E F G H$ non sia la metà dell'area del rettangolo $A B C D$.

- La seconda mossa è del verificatore che muovendo i punti $G$ e $H$ deve fare in modo che l'area $E F G H$ sia la metà dell'area del rettangolo $A B C D$.

- Quando il verificatore ha concluso la sua mossa il falsificatore clicca su TEST per verificare se il verificatore ha raggiungo l'obiettivo. In caso affermativo, segnate, nella tabella sottostante, un punto al verificatore. In caso contrario, segnate il punto al falsificatore.

- Cliccate sU TEST per nascondere il valore dell'area di EFGH e iniziate una nuova sfida (scambiatevi i ruoli e gli obiettivi ogni due partite e giocate un totale di otto partite).

Come messo in luce dalle regole del gioco, l'esito della sfida viene stabilito in base al 
valore dell'area di $E F G H$ visualizzabile cliccando su TEST.

Terminata la fase di gioco inizia la fase di indagine guidata dalle seguenti domande stimolo:

1. Qual è la strategia vincente per il verificatore? Ossia: quale condizione geometrica permette al verificatore di raggiungere l'obiettivo qualunque sia la mossa del falsificatore?

2. Qual è il teorema che avete scoperto? Formulate un enunciato nella forma "Se...allora..."

3. Scrivete le ipotesi, la tesi e dimostratelo.

4. La condizione che avete individuato come ipotesi è solo sufficiente o è anche NECESSARIA?

Per rispondere, formulate il teorema inverso, ossia il teorema che si ottiene invertendo ipotesi e tesi:

5. Adesso, giocando altre partite, cercate di stabilire se il teorema inverso è vero o falso. Scrivete le vostre osservazioni.

6. FACOLTATIVO: Se avete concluso che il teorema inverso è vero, dimostratelo. Se avete concluso che è falso, fornite un contro esempio.

7. Se al posto del rettangolo $A B C D$ ci fosse un parallelogramma, il teorema che avete scoperto sarebbe ancora valido? Scrivete le vostre osservazioni.

Partendo dall'esperienza di gioco, le domande intendono condurre gli studenti alla scoperta del teorema su cui è basato il gioco. In particolare, la domanda 1 richiede l'esplicitazione della condizione necessaria e sufficiente a garantire che l'area di $E F G H$ sia la metà dell'area di $A B C D$. Tale condizione può essere espressa in vari modi:

- una diagonale di $E F G H$ deve essere parallela ad un lato di $A B C D$;

- una diagonale di $E F G H$ deve essere perpendicolare ad un lato di $A B C D$;

- due vertici di $E F G H$ devono essere simmetrici rispetto ad un asse di simmetria di $A B C D$.

Gli studenti, inizialmente, potrebbero individuare una condizione sufficiente ma non necessaria, ad esempio:

- entrambe le diagonali di $E F G H$ devono essere parallele/perpendicolari ai lati di $A B C D$.

La domanda 2 prevede la riformulazione di quanto scoperto utilizzando il connettivo logico "Se ... allora...". Per rispondere a questa domanda gli studenti dovrebbero inserire come ipotesi la condizione geometrica individuata come risposta alla domanda 1 e come tesi la relazione tra le aree che si osserva ogni volta in cui si verifica tale condizione. Una possibile formulazione potrebbe essere:

- Se una diagonale del quadrilatero $E F G H$ inscritto al rettangolo $A B C D$ è parallela ad un lato del rettangolo allora l'area di $E F G H$ è la metà dell'area di $A B C D$.

Questa domanda intende creare un distacco dalla situazione empirica del gioco e guidare gli studenti verso una generalizzazione teorica.

Una volta formulato il teorema, la domanda 3 richiede la sua dimostrazione. Riportiamo in Figura 3 una possibile dimostrazione che gli studenti coinvolti nella sperimentazione potrebbero produrre. 
Figura 3

Possibile risposta alla domanda 3 .

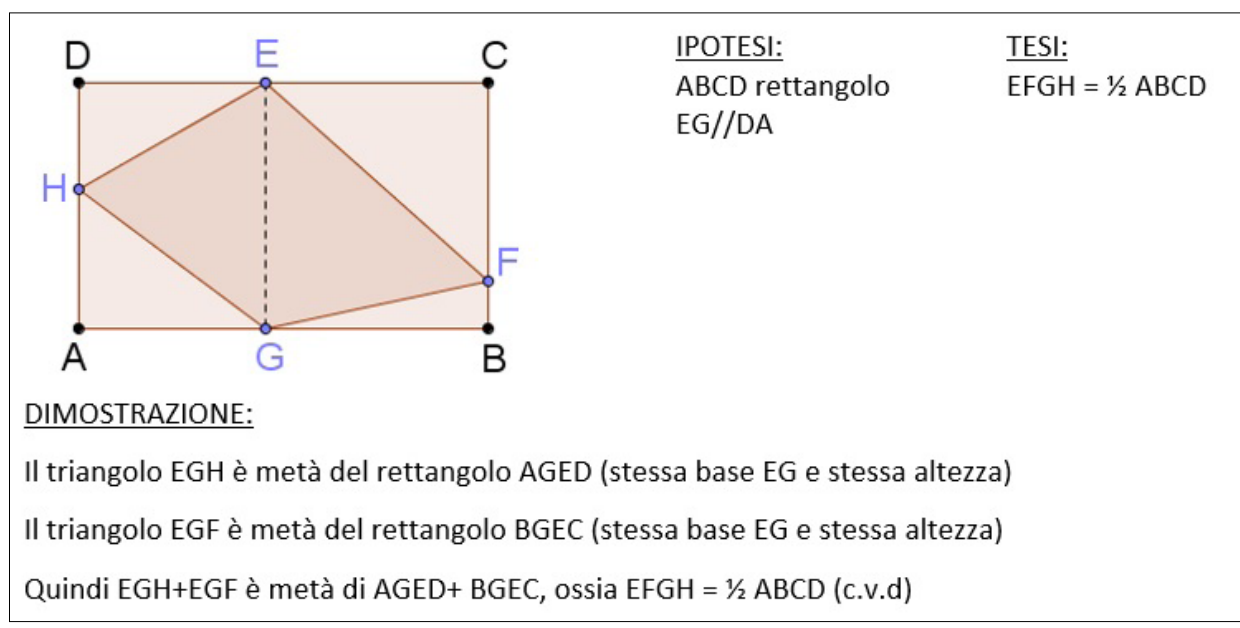

Le successive tre domande riguardano il teorema inverso: innanzitutto viene richiesta la sua formulazione, successivamente la verifica empirica della sua verità utilizzando il gioco ed infine la sua dimostrazione. Per quanto riguarda la formulazione, basterà che gli studenti scambino ipotesi e tesi del teorema formulato come risposta alla domanda 3. Per stabilire la sua verità usando il gioco, gli studenti potrebbero ragionare sulla contronominale e quindi osservare che mettendo le diagonali di $E F G H$ non parallele ai lati di $A B C D$ l'area di $E F G H$ non è mai la metà di $A B C D$ oppure provare ad ottenere la relazione tra le aree senza la condizione sulle diagonali ed osservare che ciò è impossibile. La dimostrazione del teorema inverso non è semplice, pertanto in fase di sperimentazione l'abbiamo lasciata facoltativa. È possibile procedere per assurdo in due diversi modi: sfruttando l'equiscomponibilità delle figure o usando il teorema di Talete. Infine, I'ultima domanda richiede una generalizzazione del teorema scoperto indebolendo l'ipotesi riguardante il tipo di quadrilatero $A B C D$ in oggetto. Se il teorema viene formulato usando la condizione di parallelismo tra una diagonale di $E F G H$ e un lato di $A B C D$, allora è possibile generalizzarlo per i parallelogrammi. II medesimo risultato non può essere ottenuto se viene usata la condizione di perpendicolarità.

La progettazione di questa attività didattica prevede quindi la scoperta, la formulazione e la dimostrazione di un primo teorema, successivamente la formulazione, la verifica e la dimostrazione del teorema inverso ed infine la generalizzazione dei due teoremi attraverso l'indebolimento delle sue ipotesi. Con questa attività gli studenti avranno la possibilità di scoprire tre teoremi veri e, nella discussione di classe, di riflettere sul perché per un matematico un teorema non è bello e non è finito fin quando non viene espresso nella terza formulazione.

\section{Analisi delle produzioni degli studenti}

La coppia videoregistrata è composta da Viola e Andrea ${ }^{2}$. Entrambi avevano partecipato alla sperimentazione l'anno precedente lavorando insieme. Dopo aver giocato per circa venti minuti ed essersi sfidati in sette partite, tutte vinte dal falsificatore

2. Per la tutela della privacy degli studenti coinvolti, i nomi sono stati cambiati. 
tranne I'ultima, Viola e Andrea, si accingono a sfidarsi per l'ultima volta. Andrea è il verificatore e ha prodotto la configurazione vincente di Figura 4 ma non ne è consapevole. Viola, il falsificatore, nota la configurazione vincente e incoraggia Andrea a premere il tasto TEST per accertare la vittoria.

1. V.: «Vai vai! È giusto [guardando la Figura 4]!»

2. A.: «Sono il migliore due volte!»

3. V.: «Vuoi sapere perché è giusto?»

4. A.: «Mm...»

5. V.: "Questo qua lo vedi [indicando il triangolo rettangolo $A F B$, Figura 4]? Due rettangoli... se tracci una linea [traccia metaforicamente $F H$ con la penna] ne viene un altro uguale... tracciando la linea si vede che questo qui [indicando $H C D$ ] è uguale a questo qui [indicando $D F H$ ]. Quindi per forza devono essere uguali».

Figura 4

Configurazione vincente prodotta da Andrea.

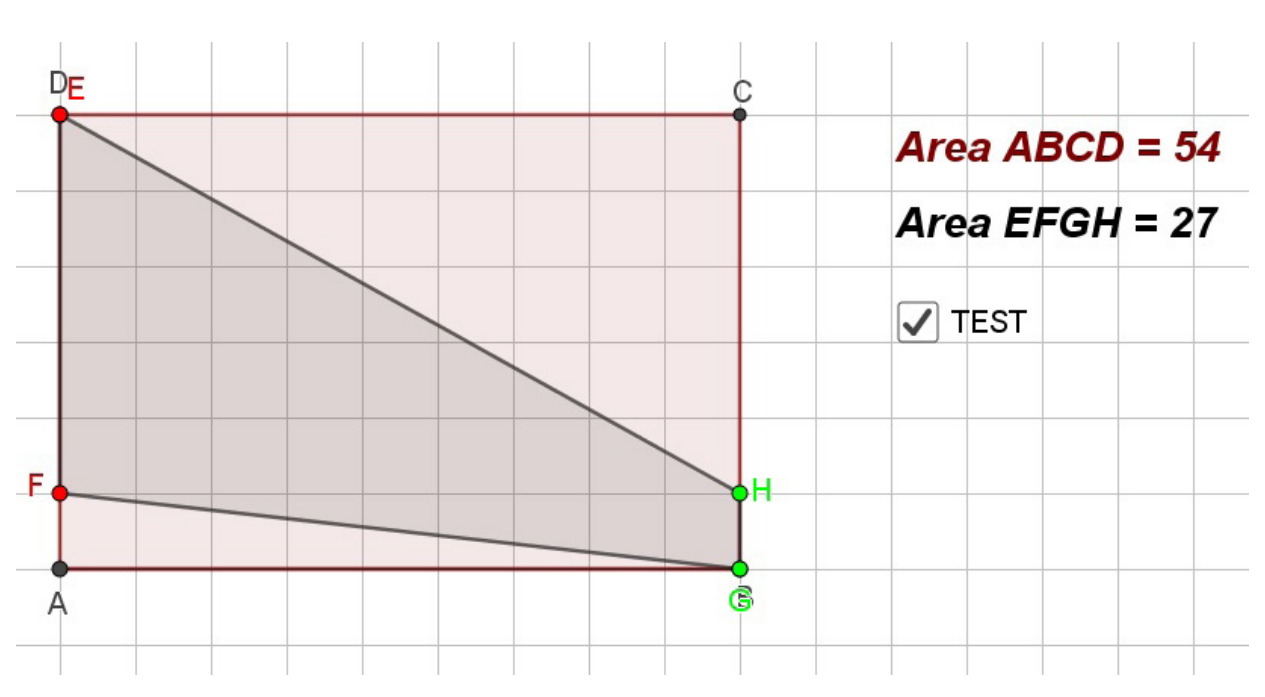

Dalla trascrizione si nota che Viola non ha ancora scoperto la strategia vincente del verificatore, ossia la condizione che permette al verificatore di vincere qualsiasi sia la configurazione prodotta dal falsificatore. Nonostante ciò, il parallelismo della diagonale $F H$ rispetto al lato $A B$ viene usato implicitamente per dimostrare la congruenza tra le coppie di triangoli $A F B$ e $B H F, C D H$ e $D F H$. Viola capisce che nella Figura 4 I'area di $E F G H$ è la metà dell'area di $A B C D$ (intervento 1) e attivando un ragionamento di tipo regressivo, produce una dimostrazione che pur non essendo rigorosa e completa permette di mostrare perché si verifica tale relazione tra le aree delle due figure. Qualche minuto dopo Viola formula una prima congettura circa la condizione geometrica che permette al verificatore di ottenere la relazione tra le aree:

Congettura 1: «EF e $G H$ devono essere paralleli ai lati del rettangolo».

La congettura, che implica che $E F G H$ sia un trapezio, non è corretta. Probabilmente è frutto di una generalizzazione della Figura 4 che non tiene in considerazione tutte le configurazioni che gli studenti hanno osservato in precedenza. È possibile ottenere questa configurazione solo se il falsificatore porta $E$ o $F$ a coincidere con un vertice del rettangolo. Nel formulare la congettura 1, Viola compie un ulteriore passo indietro nel ragionamento, perché dalla dimostrazione passa alla formulazione dell'ipotesi del teorema.

Viola, dopo aver formulato la congettura, sposta il punto $E$ sul lato $D C$ come illustrato in Figura 5, lasciando visibile il valore dell'area di $E F G H$ e si accorge che la congettura da lei formulata era errata. 
6. V.: «Però così non è cambiato niente (osservando la Figura 5, ottenuta dopo aver spostato $E$ ). Allora non c'entra niente che devono essere paralleli ai lati. Guarda se metti così è sempre 27 l'area però non sono paralleli».

Figura 5

Controesempio alla congettura 1.
Figura 6

Condizione né necessaria né sufficiente affinché

I'area di $E F G H$ pari alla metà dell'area di $A B C D$.

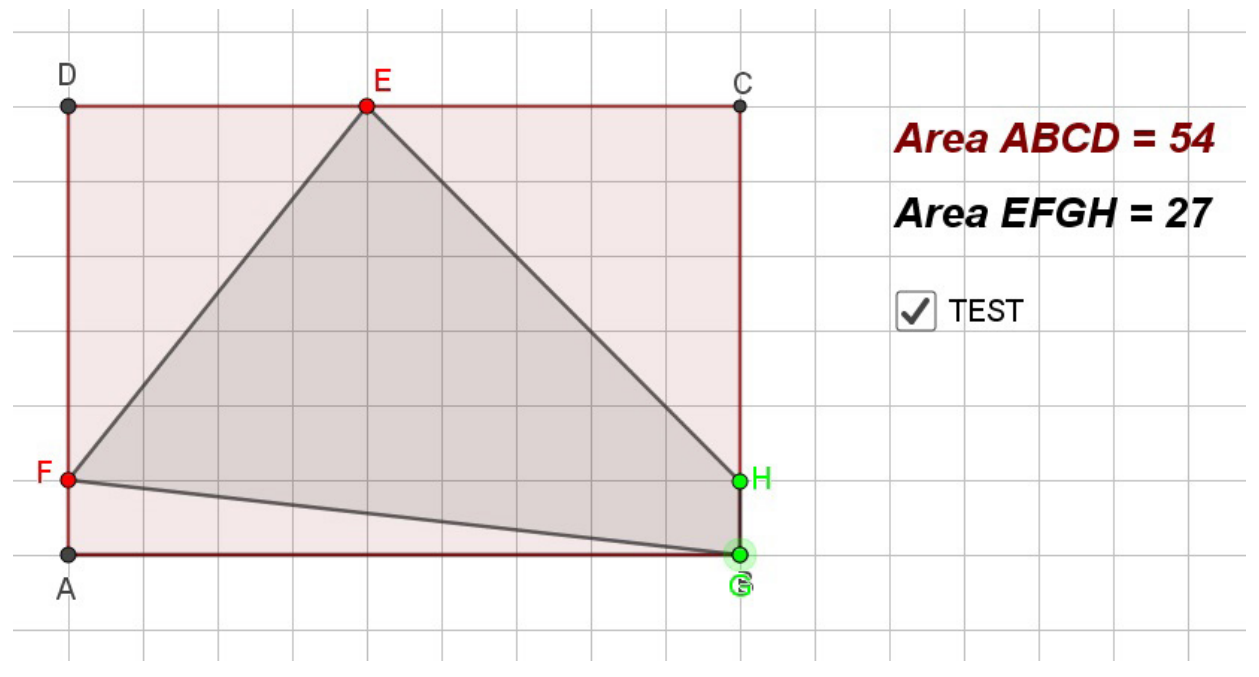

Viola, spostando il punto $E$, ottiene la configurazione di Figura 5 che rappresenta un controesempio alla congettura 1 da lei formulata. Si tratta infatti di una configurazione in cui la tesi riguardante la relazione tra le aree è soddisfatta, ma l'ipotesi di parallelismo di una coppia di lati opposti del quadrilatero $E F G H$ non è verificata. Qualche minuto dopo, in seguito ad un'esplorazione con il gioco in cui produce la configurazione illustrata in Figura 6, Viola formula un'altra congettura.

Congettura 2: «Se fai le diagonali perpendicolari ti viene».

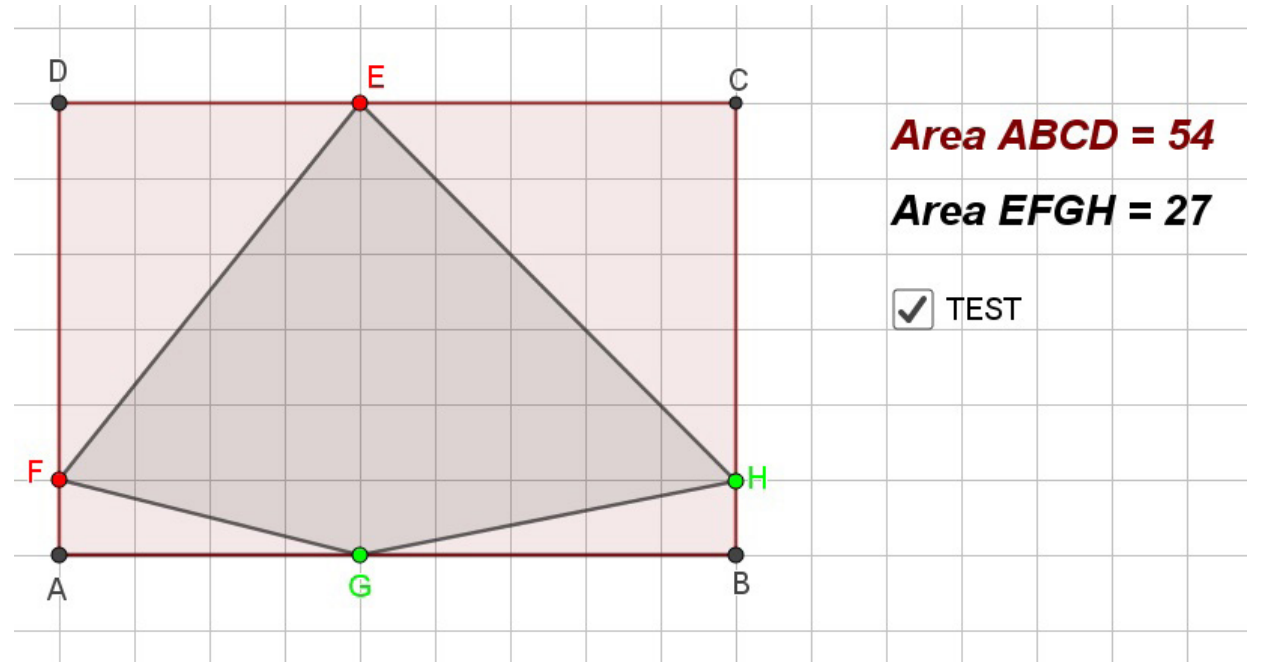

L'osservazione di Viola non è corretta, la condizione di perpendicolarità delle diagonali non è né necessaria né sufficiente. Si tratta di un esempio lampante in cui l'aspetto "figurale" può interferire con l'aspetto "concettuale". È vero che le diagonali sono perpendicolari ma questo è una conseguenza del fatto che sono parallele ai lati del rettangolo. Quindi la "vera" condizione è quella di parallelismo con almeno 
uno dei lati del rettangolo e non di perpendicolarità tra le diagonali, pertanto la congettura 2 è errata.

A questo punto Viola e Andrea fanno un'altra osservazione relativamente alla perpendicolarità delle diagonali. Nel file GeoGebra costruiscono una diagonale e poi con il comando "retta perpendicolare" costruiscono una retta passante per $E$ perpendicolare alla diagonale $\mathrm{FH}$ e si accorgono che questa retta non coincide con la diagonale $E G$, come mostrato dalla Figura 7.

Figura 7

La condizione di perpendicolarità non è necessaria affinché l'area sia la metà.

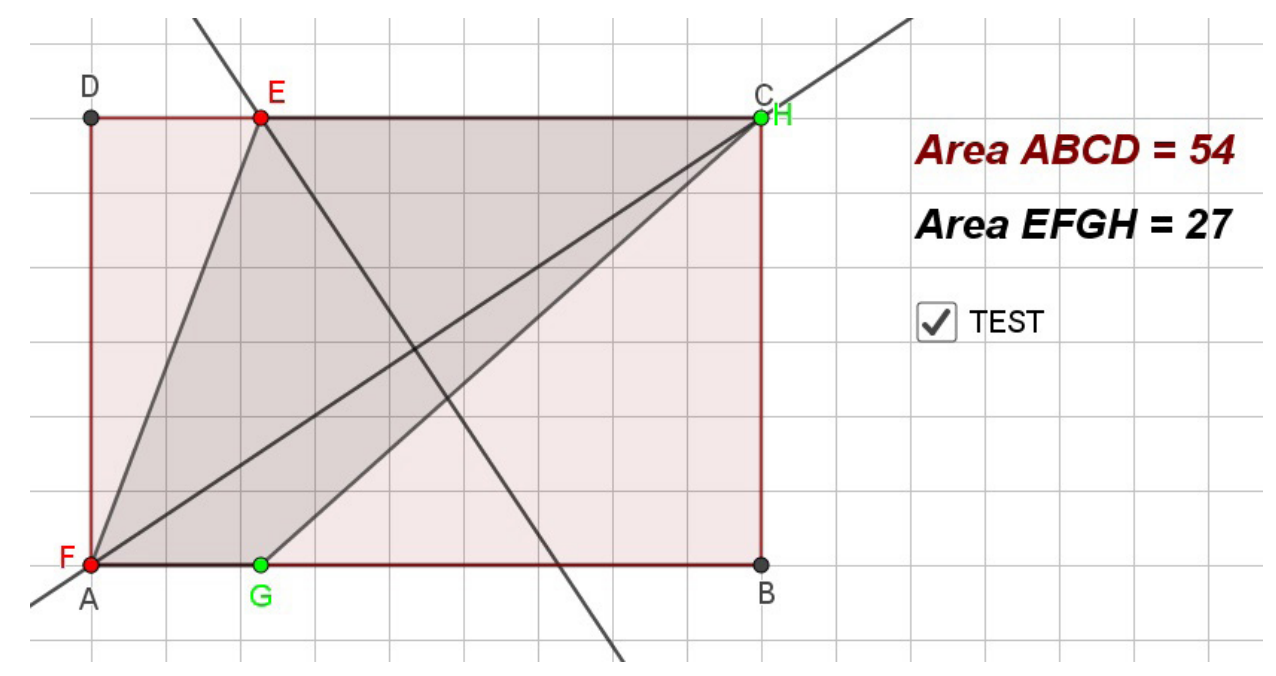

Per verificare che la perpendicolarità delle diagonali non è una condizione necessaria, Viola ha rovesciato il suo ragionamento che da regressivo diventa progressivo. Si noti che rispetto alla congettura 2 (la perpendicolarità come condizione sufficiente) Viola ed Andrea non producono un vero e proprio controesempio.

A questo punto, Viola e Andrea riattivano il ragionamento regressivo e, osservando la Figura 8, formulano una nuova strategia.

Congettura 3: «Se due punti opposti sono sulla stessa retta allora l'area del trapezio è metà dell'area del rettangolo».

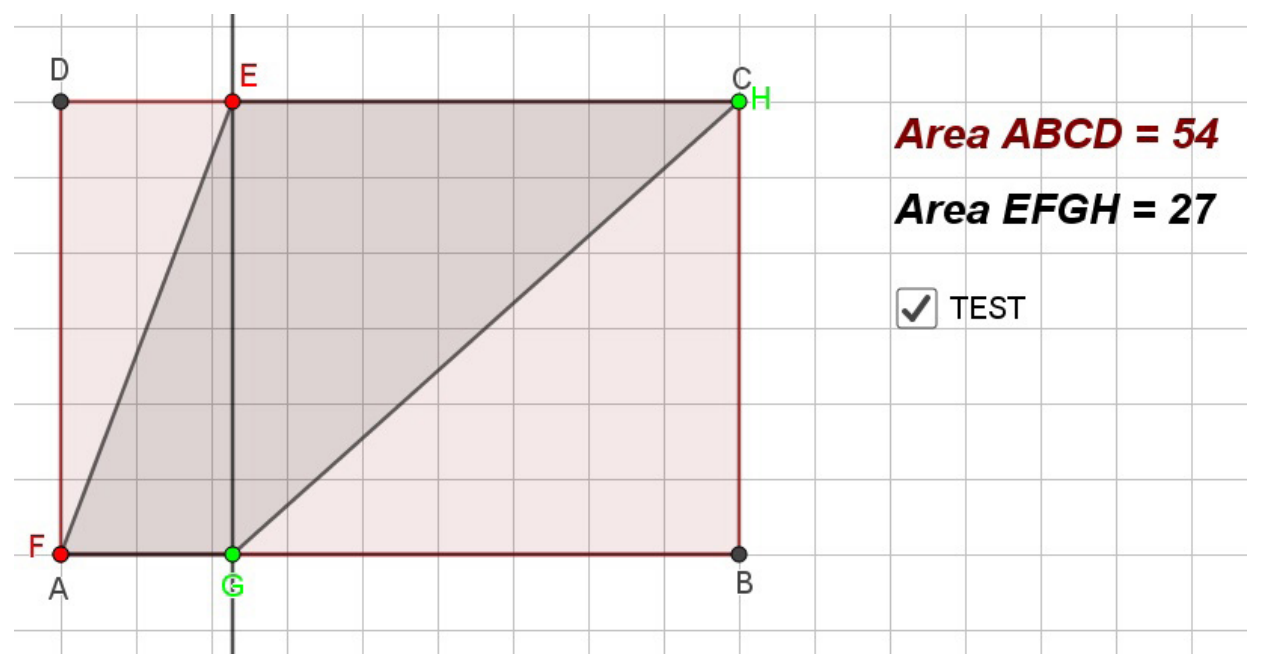


La congettura 3 non è ancora espressa in linguaggio matematico chiaro e corretto, ma mostra che gli studenti stanno focalizzando la loro attenzione sulla diagonale $E G$, elemento su cui si fonda la condizione necessaria e sufficiente per ottenere la relazione tra le aree desiderata. II ragionamento attivato nel produrre questa nuova congettura è nuovamente di tipo regressivo, dalla tesi all'ipotesi.

Al momento della formulazione della congettura 3, gli studenti fanno riferimento unicamente alla configurazione del trapezio. Viola ed Andrea attivano un ragionamento per casi: in generale la strategia vincente consiste nel fare le diagonali perpendicolari, tranne nel caso della configurazione trapezio in cui «due punti opposti stanno sulla stessa retta».

7. V.: «Però anche così però viene [guardando la Figura 9]!»

8. A.: «Eh?»

9. V.: «Anche così viene bene... Quando lo dividi viene uguale. Vedi questo triangolo rettangolo qua [indicando $C E H$ ] ? Se ci tracci un'altra linea viene un triangolo rettangolo uguale [tracciando metaforicamente una retta passante per $H$ e parallela a $C D$ ]... E poi c'è questo qui [indicando $G B H$ ], se ci tracciassi una linea [riferendosi alla retta passante per $H \mathrm{e}$ parallela a $C D$ ] viene così e sono uguali».

10. A.: "Sì. [Viola muove prima $H$ e poi $F$, lasciano $E G$ parallelo ad $A D$ ] Abbiamo trovato $E$ e $G$ e quindi ora se muovi $F$ e $H \ldots$ indipendentemente da cosa muovi rimane sempre $27 \ldots E$ e $G$ devono star lì.

11. V.: «E ma allora non tutti i punti devono essere allineati solo $E$ e $G \ldots$ Mentre se proviamo ad allineare questi due [allinea $F H$ e muove $G] \ldots$ Basta che due punti siano allineati».

Figura 9

Nuova configurazione vincente.
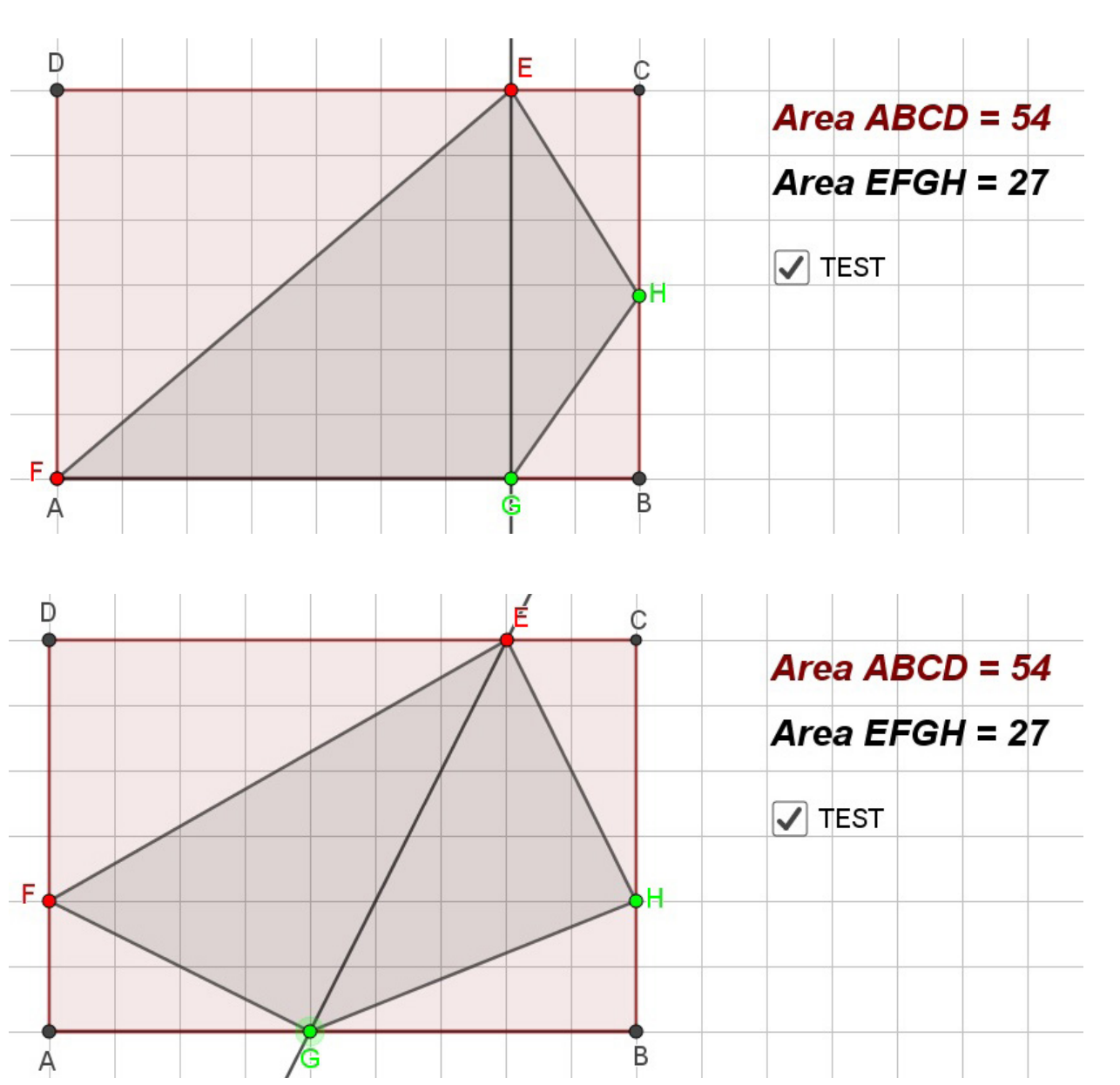
Gli studenti producono una nuova configurazione vincente che non rientra nei casi precedenti perché non è un trapezio e non ha le diagonali perpendicolari. Una volta scoperta questa configurazione vincente, Viola riattiva il ragionamento regressivo perché dall'osservazione della tesi passa alla dimostrazione a parole che l'area di $A B C D$ è il doppio dell'area di $E F G H$ usando l'equiscomponibilità (intervento 9). Terminata la dimostrazione, Viola inizia a muovere prima $F$ e poi $H$, lasciando invariate le posizioni di $E$ e $G$, intanto Andrea generalizza quanto osserva (intervento 10). Viola completa la generalizzazione iniziata da Andrea considerando l'altra diagonale di $E F G H$ : mette $F H$ parallelo ad $A B$ (vedere Figura 10) e inizia a muovere $G$.

Gli studenti concludono quindi che «Basta che due punti siano allineati», congettura già individuata precedentemente ma che pensavano valesse solo per le configurazioni in cui $E F G H$ era un trapezio.

Una volta generalizzata la congettura, Viola e Andrea esplorano il caso degenere in cui $E F G H$ è un triangolo e constatano che la relazione tra le aree continua a valere dimostrando che l'area del rettangolo $A B C D$ è il doppio dell'area del triangolo di uguale base a altezza.

Figura 11

Esplorazione di un caso degenere.

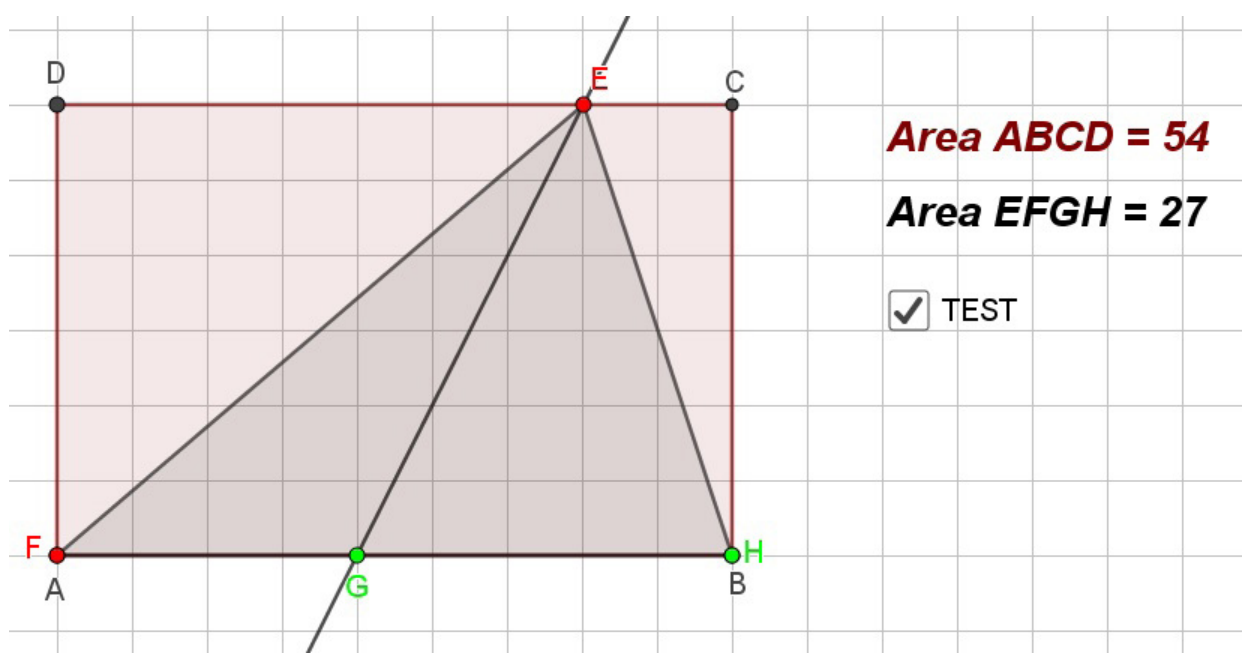

Al termine di questa esplorazione gli studenti specificano ciò che era rimasto poco chiaro nella congettura 3: "Sulla stessa retta vuol dire che $E G$ è parallelo ad $A D$ ». Dopo circa un'ora di gioco ed indagine gli studenti esprimono la congettura corretta usando un linguaggio matematico appropriato.

\section{6 concusioni}

La Figura 12 riassume il processo di scoperta della condizione necessaria e sufficiente su cui è basato il gioco. In ciascun riquadro è riportato il minuto in cui Viola e Andrea iniziano il processo descritto e l'eventuale configurazione prodotta. La freccia rivolta a destra indica che il ragionamento che permette il passaggio tra i due processi descritti nei riquadri è di tipo progressivo, mentre la freccia rivolta a sinistra indica un ragionamento di tipo regressivo. I riquadri sono disposti su quattro righe che corrispondono alle quattro congetture formulate (congettura 1,2,3 e la generalizzazione della congettura 3). Ciascuna riga deve essere letta da sinistra a destra. 


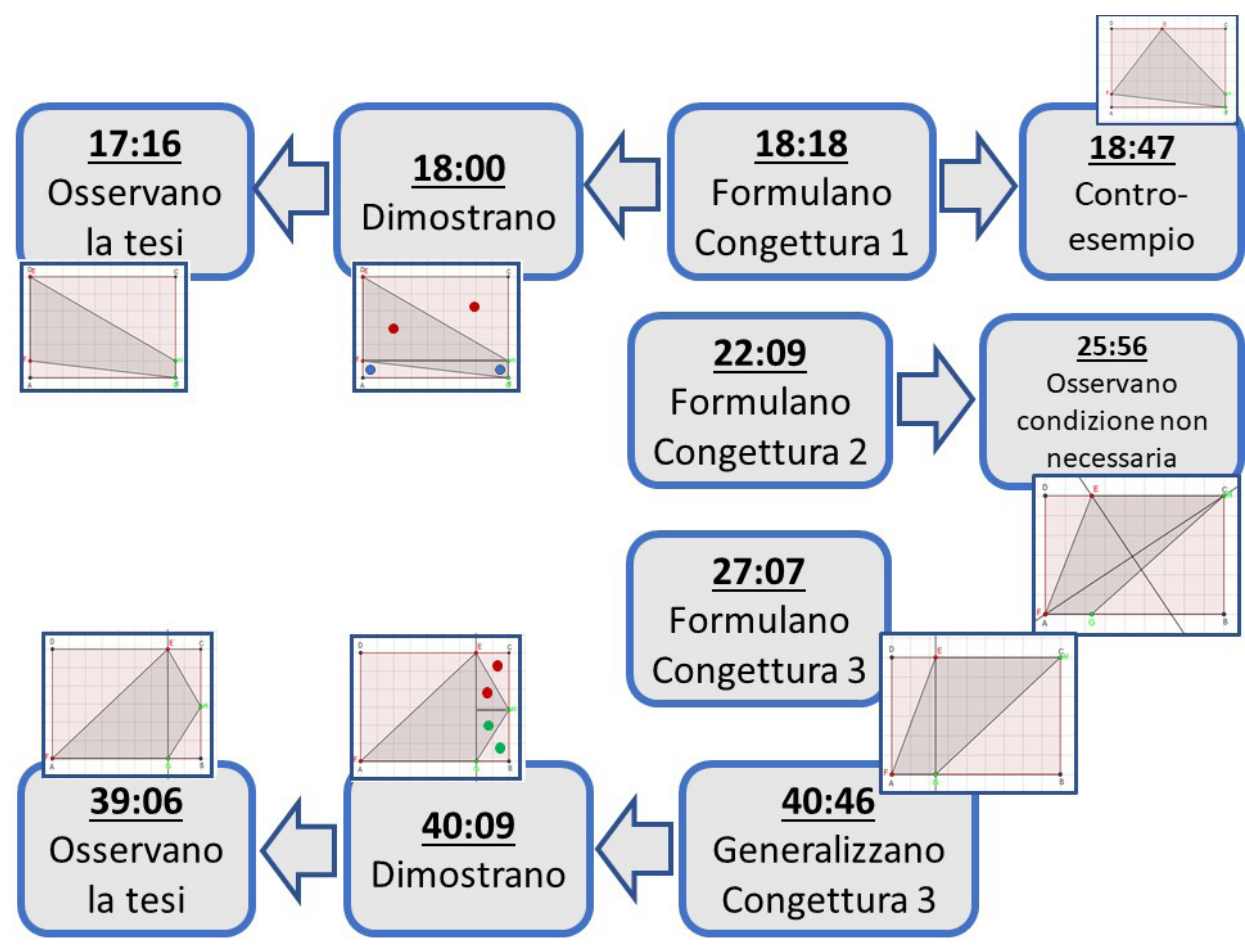

Partendo dall'osservazione della relazione tra le aree (minuto 17:16) gli studenti compiono un passo indietro nel ragionamento e dimostrano la relazione (minuto 18:00). II loro processo di pensiero segue un ordine inverso rispetto a quello seguito dal processo deduttivo indicato dalla direzione della freccia. Dopodiché compiono un ulteriore passo indietro e formulano una prima congettura che qualche secondo dopo confutano con un controesempio. Al minuto 22:09 formulano una seconda congettura che rappresenta una errata concettualizzazione della situazione osservata. Si accorgono che questa congettura non permette di spiegare tutti i tipi di configurazione osservati nel gioco, ad esempio il caso del trapezio. Al minuto 27:07 formulano una terza congettura che prende in considerazione l'elemento geometrico corretto, ossia una diagonale di $E F G H$ ma che non è ancora espressa in linguaggio matematico chiaro e corretto (non si parla esplicitamente di parallelismo della diagonale di $E F G H$ e del lato di $A B C D$ ). La scoperta di una nuova configurazione che non rientra nei casi trattati precedentemente li porta a reiterare il ciclo: al minuto 40:09 ritornano sulla dimostrazione e operando un ulteriore passo indietro nel ragionamento generalizzano la terza congettura a tutti i casi possibili.

$\mathrm{Nel}$ caso descritto, è possibile riconoscere nei processi di esplorazione, indagine e dimostrazione attivati dagli studenti la logica dell'indagine in atto: il ragionamento regressivo si attiva nella formulazione di congetture, il ragionamento progressivo si manifesta nella produzione delle dimostrazioni e nella verifica delle congetture. Attraverso la produzione di controesempi le prime due congetture si rivelano errate mentre soltanto la congettura 3 permette di spiegare tutte le configurazioni vincenti. La minuziosa indagine effettuata dagli studenti ha permesso loro di "maneggiare" con gli elementi del teorema, dandogli un significato e un senso. Grazie ad essa, Viola e Andrea hanno tutti gli elementi per enunciare e dimostrare formalmente il teorema. Si tratta solo di riprendere gli elementi osservati in fase di indagine e sistemarli in corrette catene deduttive. Dopo aver riconosciuto la correttezza della congettura 3 , infatti, Viola e Andrea producono l'enunciato «Se una diagonale del quadrilatero 
$E F G H$ inscritto al rettangolo $A B C D$ è parallela ad un lato del rettangolo allora l'area di $E F G H$ è la metà dell'area di $A B C D »$, corredandolo di una dimostrazione scritta senza difficoltà.

Dall'analisi di questa attività appare dunque plausibile che il processo della logica dell'indagine supporti efficacemente la continuità cognitiva (Garuti, Boero, Lemut \& Mariotti, 1996) tra fase di esplorazione e fase di dimostrazione.

È importante osservare che con questo tipo di attività gli studenti hanno usato GeoGebra lavorando sia sul piano empirico che sul piano teorico nel momento in cui formulano argomentazioni e dimostrano quanto osservato empiricamente. L'ambiente di geometria dinamica con all'interno le dinamiche del gioco semantico alla Hintikka sembra quindi supportare gli studenti nel passaggio al piano teorico, che tante volte rischia di restare appiattito dall'evidenza empirica.

\section{Bibliografia}

Arsac, G. (1999). Variations et variables de la démostration géométriques. Recherches en Didactique de Mathématiques, 19(3), 357-390.

Antonini, S., \& Baccaglini-Frank, A. (2015). II trascinamento di mantenimento nella formulazione di congetture in ambienti di geometria dinamica. L'Insegnamento della Matematica e delle Scienze Integrate, 38 A-B.3, 257-278.

Barbero, M., \& Gómez-Chacón, I.M. (2018). Analysing regressive reasoning at university level. In V. Durand-Guerrier, R. Hochmuth, S. Goodchild \& N. M. Hogstad (Eds.), Proceeding of INDRUM 2018 (pp. 204-213), University of Agder: Kristiansand, Norway.

Brousseau, G. (1997). Theory of didactical situations in mathematics. Dordrecht: Kluwer Academic Publishers.

MIUR. (2007) Decreto ministeriale n. 139, 22 agosto 2007. Roma: Ministero Istruzione Università e Ricerca. Disponibile in https://archivio.pubblica.istruzione.it/normativa/2007/dm13 9 07.shtml (consultato il 19.09.2019).

Fischbein, E. (1993). The theory of figural concepts. Educational studies in mathematics, 24(2), 139-162.

Garuti, R., Boero, P., Lemut, E., \& Mariotti, M.A. (1996). Challenging the traditional school approach to theorems: A hypothesis about the cognitive unity of theorems. In L. Puig \& A. Gutierrez (Eds.), Proceedings of the 20th Conference of the International Group for the Psychology of Mathematics Education (vol. 2, pp. 113-120), Valencia, Spain: PME.

Healy, L. (2000). Identifying and explaining geometrical relationship: Interactions with robust and soft Cabri constructions. In T. Nakahara \& M. Koyama (Eds.), Proceedings of the 24th Conference of the International Group for the Psychology of Mathematics Education (vol. 1, pp. 103-117), Hiroshima, Japan: PME.

Hintikka, J. (1998). The principles of mathematics revisited. Cambridge University Press.

Hintikka, J. (1999). Inquiry as inquiry: a logic of scientific discovery. Springer Science \& Business Media.

Laborde, C. (2005). Robust and soft constructions: Two sides of the use of dynamic geometry environments. In W.C. Yang (Ed.), Proceedings of the 10th Asian technology conference in mathematics (pp. 22-35), Korea: National University of Education. 
Peirce, C. S. (1960). Collected papers. In C. Hartshorne \& P. Weiss (Eds.), Harvard University Press, Cambridge (Mass.).

Soldano, C., \& Sabena, C. (2019). Semiotic potential of inquiring-game activities. In M. Graven, H. Venkat, A. A. Essien \& P. Vale (Eds.), Proceedings of 43th Conference of the International Group for the Psychology of Mathematics Education, (vol. 3, pp. 319-327), Pretoria, South Africa: PME.

\section{Autori/Carlotta Soldano ${ }^{\star}$ e Gaetano Di Caprio ${ }^{\circ}$}

*Dipartimento di Filosofia e Scienze dell’Educazione, Università degli Studi di Torino "Istituto di Istruzione Superiore "Erasmo da Rotterdam", Nichelino (Torino)

carlotta.soldano@unito.it; gaetano.dicaprio@istruzione.it 